\title{
Ipsilesional neglect: behavioural and anatomical features
}

\author{
M Kim, D L Na, G M Kim, J C Adair, K H Lee, K M Heilman
}

Department of Neurology, University of Florida College of Medicine, FL, USA M Kim

K M Heilman

Neurology Service, Department of Veterans Affairs Medical Center, Gainesville, FL, USA K M Heilman

Department of Neurology, Samsung Medical Center, Seoul, Korea

$\mathrm{D} \mathrm{L} \mathrm{Na}$

G M Kim

K H Lee

Department of Neurology, University of New Mexico, and the Neurology Service, Department of Veterans Affairs Medical Center, Albuquerque, NM, USA

J C Adair

Correspondence to: Dr Kenneth M Heilman, Department of Neurology, University of Florida College of Medicine, PO Box 100236, Gainesville, FL 32610-0236, USA. Telephone 001352392 3491; fax 001352393 6893; email

heilman@medicine.ufl.edu

Received 6 February 1998 and in revised form

5 January 1999

Accepted 28 January 1999

\begin{abstract}
Objective-To learn more about the behavioural and anatomical features of ipsilesional neglect.

Methods-Thirty consecutive patients with spatial neglect were tested on cancellation and line bisection tasks. To learn if patients with ipsilesional neglect demonstrate the sensory-attentional or motorintentional type of neglect, a video apparatus was used that dissociates these determinants.
\end{abstract}

Results-Five patients showed evidence of ipsilesional neglect. This phenomenon was seen only on the line bisection task. All patients with ipsilesional neglect had lesions involving frontal-subcortical regions. Although ipsilesional neglect evolved from early in three of five cases, the other patients displayed ipsilesional neglect without initial contralateral neglect, suggesting that ipsilesional neglect cannot be fully attributed to a compensatory strategy. The results of the tests that used the video apparatus indicate that right sided frontal or subcortical injury may induce contralateral attentional or intentional "approach" behaviours.

Conclusions-Ipsilesional neglect is most often associated with frontal-subcortical lesions, cannot be entirely attributed to a compensatory strategy, and may be induced by an attentional bias, an intentional bias, or both.

(F Neurol Neurosurg Psychiatry 1999;67:35-38)

Keywords: neglect; ipsilesional; frontal-subcortical; approach

Neglect is the failure to report, respond, or orient to stimuli that cannot be attributed to primary sensory or motor defects. ${ }^{1}$ Although different behavioural manifestations have been described, most involve disrupted processing of stimuli in the space opposite a brain lesion. However, impaired processing of stimuli ipsilateral to a hemispheric lesion has also recently been reported. ${ }^{2-4}$

Kwon and Heilman used the term "ipsilesional neglect" to refer to patients who, after right brain injury, deviated to the left on the line bisection task. ${ }^{2}$ On the cancellation task, subjects with a right sided lesion often miss targets on the right ipsilesion side. ${ }^{56}$ However, these previously reported patients omitted more contralesional targets than ipsilesional targets when performing the cancellation task. Because their error pattern was not spatially reversed, these patients were not considered to have ipsilesional neglect.

The phenomenon of ipsilesional neglect has been reported only rarely. Therefore, the anatomical features and neuropsychological mechanisms remain unclear. We assessed consecutive patients with right hemispheric injury for ipsilesional neglect. We attempted to define the anatomical loci of lesions associated with this disorder. Neglect can be caused by sensory-attentional and motor intentional deficits or biases. In this study, we attempted to learn if ipsilesional neglect is caused by an attentional deficit, an intentional deficit, or both. Lastly, Robertson et al suggest that ipsilesional neglect may be related to a compensatory left sided scanning strategy. If this postulate is correct, we would expect that ipsilesional neglect would be seen only after an initial period of contralesional neglect.

\section{Methods}

Between January 1995 and March 1996, 53 consecutive patients with non-lacunar right hemispheric stroke were evaluated at Samsung Medical Centre in Seoul, South Korea. Eleven patients were not testable because of depressed sensorium. The remaining 42 patients were screened with line bisection and cancellation tasks, and 30 patients with neglect were identified. All patients underwent a standard neurological examination as well as brain imaging with either CT or MRI. To assess and quantify neglect phenomena, the examiners administered additional cancellation and line bisection tasks. Repeated observations over several weeks were available on four of five patients.

To dissociate perceptual-attentional from action-intentional determinants of performance, patients performed bisection tasks using a video apparatus that dissociates these determinants. ${ }^{7}$ In brief, subjects performed tasks with their ipsilesional hand. They could not see this hand directly, but viewed the hand and lines through a closed circuit TV. In the direct condition, movements on the stimulus page were spatially congruent with those depicted on the monitor. In the indirect condition, the left side of the stimulus projects to the right side of the monitor and vice versa. Patients were separated into perceptualattentional or premotor-intentional types by comparing performance between the direct and indirect conditions. For example, in the indirect or reversed view conditions, reversal of mean line bisection bias from the left to the right side of the stimulus page indicates a primarily attention-perceptual processing defect for the right side of the monitor or a bias to 
Table 1 Patients with ipsilateral neglect

\begin{tabular}{|c|c|c|c|c|c|}
\hline Patient & Age/sex & Initial neurological finding & Lesion & $\begin{array}{l}\text { Poststroke } \\
\text { interval }\end{array}$ & $\begin{array}{l}\text { Neglect } \\
\text { type }\end{array}$ \\
\hline 1 & $55 / \mathrm{F}$ & $\begin{array}{l}\text { hemiplegia, sensory loss, anosognosia, } \\
\text { asomatognosia }\end{array}$ & Frontal basal ganglia & 24 dayst & ATT \\
\hline & & & & 3 months $\dagger$ & \\
\hline 2 & $62 / \mathrm{M}$ & hemiplegia & Basal ganglia & 7 days & INT \\
\hline 3 & $63 / \mathrm{F}$ & $\begin{array}{l}\text { hemiparesis, tingling sense but no } \\
\text { objective sensory loss in left extremity }\end{array}$ & Thalamus & 17 days & ATT \\
\hline 4 & $42 / \mathrm{F}$ & hemiplegia, sensory loss & Basal ganglia & 3 days & ATT \\
\hline 5 & $55 / \mathrm{F}$ & $\begin{array}{l}\text { hemiplegia, anosognosia, } \\
\text { asomatognosia }\end{array}$ & Basal ganglia & 25 days & INT \\
\hline
\end{tabular}

${ }^{\star}$ ATT $=$ Perceptuo-attentional type of neglect, INT= premotor-intentional type of neglect.

tWhen tested at 24 days after the stroke, the patient had ATT neglect. However, when tested at 3 months poststroke, the patient had INT neglect.

attend to the right side of the monitor. By contrast, persistent bias toward the left side of the stimulus, even in the indirect or reversed feedback condition, indicates a leftward action bias.

The lines for bisection were $235 \mathrm{~mm}$ in length and $1.5 \mathrm{~mm}$ thick. Lines were centred on a white A4 size paper $(297 \times 210 \mathrm{~mm})$. The objective midpoint of lines on the table and the monitor were aligned with the midsagittal plane of the subject's trunk. Deviations from objective midpoint were measured to the nearest millimeter; a positive value denotes rightward deviation and a negative value denotes leftward deviation. A modified version of Albert's test ${ }^{6}$ was used for target cancellation. Forty black lines (25 mm length, $0.5 \mathrm{~mm}$ thick) of various orientations were dispersed in a random array on a $297 \times 210 \mathrm{~mm}$ sheet of white paper. There were 18 lines on each side of the page and four lines in the centre that were not included in the data analysis. Subjects performed 10 line bisections and one to three cancellation tasks in both the direct and indirect conditions.

\section{Results}

For the line bisection task, neglect was defined as a mean error to the right or to the left of the true midpoint that exceeded the 95\% confidence intervals (95\% CIs) for the bisection errors of control subjects. ${ }^{7}$ Neglect on the cancellation task was defined by the failure to cross out one or more targets. Based on the definitions, we found 30 subjects with neglect. Ipsilesional neglect on the line bisection task was defined by a mean magnitude of error to the side opposite the brain lesion that exceeded the $95 \%$ CI of control subjects. ${ }^{7}$ Based on this definition, five of 30 patients $(16 \%)$ had ipsilesional neglect. For the cancellation task, we defined ipsilesional neglect as a failure to cross out more targets on the side of the paper ipsilateral to the lesion than on the side contralateral to the lesion. No patient met this criterion on the cancellation task. Therefore, ipsilateral neglect was seen only in line bisection tasks. The ipsilesional neglect group comprised one man and four women (table 1). In one case (patient 5), ipsilesional neglect clearly evolved during recovery from contralateral neglect. Patient 5 had a mean bisection error to the same side as the brain lesion that exceeded $95 \%$ CIs for control subject performance (table 2).

All five patients with ipsilesional neglect had vascular lesions involving the frontalsubcortical circuits. Lesion distribution included three restricted to the basal ganglia, one restricted to the thalamus, and one with a large lesion that involved cortical and subcortical structures. No patients with ipsilesional neglect were seen with lesions limited to the posterior association areas.

By contrast with line bisection performance, patients who demonstrated ipsilesional neglect eventually located all the targets in the cancellation task. Left sided omissions were present at those times when patients showed either minimal leftward bisection bias (patient 1 and 2) or had not yet developed ipsilesional neglect and had rightward bisection bias (patient 5). However, unlike normal subjects, every patient's search began on the right and proceeded from right to left, even after they were able to mark every target. Because the five patients with IN performed normally on the cancellation task in the direct condition, testing in the indirect condition would have little meaning and was therefore not undertaken.

Table 2 Line bisection and line cancellation on direct and indirect conditions

\begin{tabular}{|c|c|c|c|c|c|c|}
\hline Patient & $\begin{array}{l}\text { Days after } \\
\text { onset }\end{array}$ & Line bisection direct & $\begin{array}{l}\text { Control line } \\
\text { bisection } \\
\text { Direct mean }\end{array}$ & $\begin{array}{l}\text { Line bisection } \\
\text { Indirect }\end{array}$ & $\begin{array}{l}\text { Line cancellation } \\
\text { Left }(\%)\end{array}$ & $\begin{array}{l}\text { Line cancellation } \\
\text { Right }(\%)\end{array}$ \\
\hline \multirow[t]{3}{*}{1} & 17 & ND & \multirow[t]{11}{*}{$-1.9(2.6)$} & ND & 33.3 & 94.4 \\
\hline & 24 & $-3.9(3.5)$ & & $38.4(32.3)$ & 94.4 & 100.0 \\
\hline & 109 & $-12.6(3.0)$ & & $-33.2(16.4)$ & 100.0 & 100.0 \\
\hline \multirow[t]{2}{*}{2} & 20 & $-5.9(5.6)$ & & ND & 94.4 & 100.0 \\
\hline & 59 & $-10.3(2.8)$ & & $-4.7(8.4)$ & 100.0 & 100.0 \\
\hline \multirow[t]{2}{*}{3} & 17 & $-15.8(6.1)$ & & $9.1(11.5)$ & 100.0 & 100.0 \\
\hline & 93 & $-6.6(5.3)$ & & $0.7(7.2)$ & 100.0 & 100.0 \\
\hline 4 & 3 & $-10.9(3.3)$ & & $2.9(6.5)$ & 100.0 & 100.0 \\
\hline \multirow{3}{*}{5} & 15 & $12.1(19.3)$ & & ND & 0 & 94.4 \\
\hline & 23 & $3.4(20.7)$ & & $29.6(40.0)$ & 77.8 & 94.4 \\
\hline & 30 & $-19.0(12.4)$ & & $-33.2(20.0)$ & 100.0 & 100.0 \\
\hline
\end{tabular}

Values are mean $(\mathrm{SD}) ; \mathrm{ND}=$ not done. 
On the line bisection task, two patients reversed the direction of bisection bias between the direct and indirect conditions, a performance consistent with a predominant influence of a left sided perceptual-attentional bias. The remaining three patients bisected to the left of true midpoint in both direct and indirect conditions, suggesting a predominant influence of a motor-intentional, left sided bias.

\section{Discussion}

The occurrence of ipsilesional neglect is not as rare as suggested by the scarcity of reports in the literature. There are several possible reasons for the limited number of reports. Firstly, ipsilesional neglect may be task dependent. In this study, ipsilesional neglect was seen only in line bisection tasks. We did not find it on the target cancellation task but this may have been related to a ceiling effect as others have found ipsilesional neglect on various neglect tests such as target cancellation, tactile exploration, figure copying, and representational drawing. ${ }^{4}$ Secondly, ipsilesional neglect may be a stage in the recovery process that develops in the postacute phase after the initial brain injury. Initially, there may be diaschisis of the uninjured portion of the right hemisphere, and ipsilesional neglect only becomes manifest when these areas recover. Three patients with ipsilesional neglect tended to acutely show contralesional neglect on cancellation tasks and minimal line bisection error, a pattern of performance associated with frontal brain lesions. ${ }^{8}$ However, over time, the cancellation bias resolved and line bisection error shifted to the left of midpoint. Similar observations were made in previous descriptions of ipsilesional neglect. ${ }^{2}{ }^{8}$

Robertson $e t a l^{4}$ suggested that ipsilesional neglect is induced by a compensatory left sided scanning strategy. There are two findings that would not fully support the compensation hypothesis. Firstly, on the line bisection task, patients 3 and 4 never demonstrated contralateral neglect before they demonstrated ipsilesional neglect, and patient 4 was tested 3 days after his stroke. Hence, these patients had neither the impetus nor the time to acquire compensatory bias to the left. Secondly, if a left sided compensatory scanning strategy were responsible for ipsilesional neglect, we would have expected all our patients to show a predominant influence of motor-intentional factors in neglect behaviour. However, several subjects had predominantly perceptualattentional neglect.

All of the patients with ipsilesional neglect had lesions involving frontal-subcortical circuits (frontal lobe, basal ganglia, and thalamus). Frontal lobe lesions may be associated with neglect. ${ }^{10}$ The frontal lobes are part of the systems that mediate both attention and intention. That attentional neglect can be in one direction and intentional neglect in the other direction provides evidence that, although the frontal lobes contribute to both systems, these systems, at least in part, are functionally independent. ${ }^{11}$ The prevailing bias may depend on task demands. Therefore, after a frontal lesion, a subject's performance may reflect a combination of ipsilesional neglect on an intentional basis and contralesional neglect on an attentional basis. As the spatial attentional requirements are reduced, they will show ipsilesional neglect, and as the attentional requirements are increased, they will show contralesional neglect. In addition, the attentional and intentional components may have different recovery patterns, and ipsilesional intentional neglect may be one stage during the course of recovery from contralesional neglect. Our findings in patients 1,2 , and 5 support this postulate.

Cunelli et $a l^{12}$ documented dissociation of neglect behaviour using a keyboard exploration task under visual guidance and while blindfolded. Unexpectedly, one patient showed normal performance under visual guidance but displayed a left sided bias while blindfolded. According to the authors, the anomalous performance "cannot be explained by any current theories of neglect." The same report mentions a patient with left hemispheric damage with ipsilesional neglect for words but contralesional neglect on other measures of neglect such as target cancellation and figure search. According to our formulation, the first instance of dissociated behaviour would result if blindfolding somehow reduced the spatial attentional demands of the task, thereby "unmasking" an intentional bias. The second might reflect relatively increased attentional demands invoked by searching across multiple objects in an array versus the relatively reduced attentional demands of processing single words.

Villardita described another dissociation using tactile exploration of a maze after right or left hemispheric stroke. ${ }^{13}$ Patients without neglect after either right or left hemispheric injuries performed the task similarly, locating a greater proportion of items from space ipsilateral to the lesion. By contrast, right hemispheric damage with contralateral left visuospatial neglect, as determined through figure copying and cancellation tests, showed the opposite behaviour on this tactile task, locating a greater proportion of items from contralesional space. Villardita thought that in the tactile modality after elimination of vision, subjects were biased toward contralesional space in an attempt to compensate for defective visuospatial processing. Alternatively, perhaps blindfolding reduced the ipsilateral visuospatial attentional bias and the contralesional intentional bias became manifest, thereby inducing ipsilateral neglect.

Riddoch $e t a l^{14}$ provided another account for task dependent dissociations of spatial bias. After left hemispheric injury, their patient showed right sided neglect in figure copying and cancellation tasks but showed left sided neglect for reading, a dissociation reported by others. ${ }^{15}$ However, the patient also neglected rightward characters when spelling. After further experimental assessment, the authors concluded that the direction of the patient's neglect depended on whether stimuli were represented as parts of one object or as independent parts of perceptual objects. Their account 
could apply to our findings if line stimuli are processed primarily as single perceptual entities, whereas target arrays or figures are processed as multiple separate objects. The hypothesis might be tested by devising "bisection" tasks using lines composed of individual elements.

The reason that frontal lesions induce ipsilesional neglect remains unclear. Denny-Brown and Chambers ${ }^{16}$ proposed that whereas normally the frontal lobe mediates avoidance behaviour, the parietal lobe mediates approach behaviour. Therefore, investigators have posited that frontal lesions cause a release of approach behaviours. ${ }^{9}$ The frontal lobe plays an important part in directed attention and intention, and these approach behaviours may be perceptual-attentional or action-intentional. In previous studies attempting to separate perceptual from action-motor factors in contralesional neglect, lesions of the frontalsubcortical structures were most often associated with motor-intentional neglect. ${ }^{17}$ In this respect, our findings using the video apparatus are somewhat anomalous. We found two out of five patients with ipsilesional neglect with performance indicative of primarily perceptual-attentional dysfunction. This finding is most compatible with an account of ipsilesional neglect, like contralesional neglect, resulting from either attentional or intentional approach behaviours toward stimuli in contralesional space.

This study was supported by the Medical Research Service of the Department of Veterans Affairs and Samsung grant No the Department
1 Heilman KM, Watson RT, Valenstein E. Neglect and related disorders. In: Heilman KM, Valenstein E, eds. Clinical neuropsychology. 3rd ed. New York: Oxford University Press, 1993:279-336.

2 Kwon SE, Heilman KM. Ipsilateral neglect in a patient following a unilateral frontal lesion. Neurology 1991;41:2001-

3 Halligan PW, Robertson IH, Pizzamiglio L, et al. The laterality of visual neglect after right hemisphere damage. Neuropsychological Rehabilitation 1991;1:281-301.

4 Robertson IH, Halligan PW, Bergego C, et al. Right neglect following right hemisphere damage? Cortex 1994;30:199213.

5 Albert ML. A simple test of visual neglect. Neurology 1973; 23:658-64.

6 Weintraub S, Mesulam MM. Right cerebral dominance in spatial attention. Further evidence based on ipsilateral neglect. Neurology 1987;44:621-5.

$7 \mathrm{Na}$ DL, Adair JC, Williamson DJG, et al. Dissociation of sensory-attentional from motor-intentional spatial neglect [abstract]. Neurology 1995;45(suppl):290.

8 Binder J, Marshall R, Lazar R, et al. Distinct syndromes of hemineglect. Arch Neurol 1992;49:1187-94.

9 Butters CM, Rapcsak SZ Watson RT, et al. Changes in sensory inattention, directional akinesia, and release of the fixation reflex following a unilateral frontal lesion: a case report. Neuropsychologia 1988;26:533-45.

10 Heilman KM, Valenstein E. Frontal lobe neglect in man. Neurology 1972;22:660-4.

11 Watson RT, Valenstein E, Heilman KM. Thalamic neglect. Possible role of the medial thalamus and nucleus reticularis in behavior. Arch Neurol 1981;38:501-5.

12 Cubelli R, Nichelli P, Bonito V. Different patterns of dissociation in unilateral spatial neglect. Brain Cogn 1991;15: $139-59$.

13 Villardita C. Tactile exploration of space and visual neglect in brain-damaged patients. F Neurol 1987;234:292-7.

14 Riddoch MJ, Humphreys GW, Luckhurst L, et al. Paradoxical neglect; spatial representations, hemisphere-specific activation, and cueing. Cognitive Neuropsychology 1995;12: 569-604.

15 Costello A, Warrington EK. The dissociation of visuospatial neglect and neglect dyslexia. $\mathcal{F}$ Neurol Neurosurg Psychiatry 1987;50:1110-6.

16 Denny-Brown D, Chambers RA. The parietal lobe and behavior. Association for Research in Nervous and Mental Diseases Proceedings 1958;36:35-117.

17 Tegner R, Levander M. Through a looking glass: a new technique to demonstrate directional hypokinesis in unilateral neglect. Brain 1991;114:1943-51. 